\title{
Estudio Psicométrico y Predicción de la Importancia de la Educación Física a Partir de las Orientaciones de Meta ("Perception of Success Questionnaire - POSQ")
}

\author{
Psychometric Study and the Prediction of the Importance of Physical Education \\ from Goal Guidance ("Perception of Success Questionnaire - POSQ")
}

\author{
Antonio Granero-Gallegos ${ }^{*}, a$, Antonio Baena-Extremera ${ }^{b}$, Manuel Gomez-Lopez $^{b}$ \\ \& J. Arturo Abraldes ${ }^{b}$ \\ ${ }^{a}$ Centro del Profesorado Cuevas-Olula, Almería, Província de Almería, España \\ $\&{ }^{b}$ Universidad de Murcia, Murcia, Província de Múrcia, España
}

\begin{abstract}
Resumen
El objetivo de esta investigación fue doble: (a) analizar las propiedades psicométricas del Perception of Success Questionnaire (POSQ) adaptado a la Educación Física (EF); y (b) analizar la predicción de la importancia y utilidad concedida a la EF a partir de las orientaciones de meta, percepción de competencia y satisfacción del alumnado. El cuestionario estuvo compuesto por los siguientes instrumentos: POSQ, Escala de Necesidades Psicológicas Básicas en el Ejercicio, Satisfacción con la EF e Importancia de la EF. Se administró a 846 estudiantes españoles de secundaria de 12 a 19 años. La estructura del POSQ se analizó mediante procedimientos confirmatorios y mostró una consistencia interna fiable y una estructura factorial estable (apoyando el modelo teórico hipotetizado: orientación al ego y a la tarea). Así, se considera un instrumento válido para medir la orientación de meta disposicional en EF. Para la predicción de la importancia de la EF a partir de las orientaciones de meta, competencia y satisfacción, se halló un modelo de ecuaciones estructurales que confirmó que la orientación a la tarea es la que más influye en la percepción de competencia y ésta en la satisfacción/diversión, lo que predice que el alumnado considere más importante y útil la asignatura. Palabras clave: Educación Física, orientación de meta, propiedades psicométricas, POSQ, autodeterminación, competencia, satisfacción/diversión.
\end{abstract}

\begin{abstract}
The present research has two main goals: (a) to analyze psychometric properties of the Perception of Success Questionnaire (POSQ) adapted to Physical Education (PE), and (b) to analyze the prediction of importance and usefulness granted to $\mathrm{PE}$ from goal orientations, perceived competence and student satisfaction. The questionnaire consisted of the following instruments: POSQ, Basic Psychological Needs in Exercise Scale, Satisfaction with PE and the Importance of PE. They were administered to 846 Spanish high school students aged 12 to 19 years. POSQ structure was analyzed by confirmatory procedures and it showed reliable internal consistency and a stable factor structure which supports the hypothesized theoretical model: ego and task orientation. Thus, it is considered a valid instrument to measure dispositional goal orientation in PE. For predicting the importance of PE from goal orientations, competence and satisfaction, it was used a structural equation model which confirmed that task orientation is the most influential on perceived competence, and the latter in satisfaction/ fun. Fact that predicts the students consider the subject more important and useful.

Keywords: Physical Education, goal orientation, psychometric properties, POSQ, self-determination, competence, satisfaction/fun.
\end{abstract}

En la actualidad, son varios los problemas que preocupan a los docentes de Educación Física (EF), por un lado el incrementar la motivación del alumnado en pro de conseguir una mayor adherencia a la práctica físico-deportiva,

* Dirección postal: Facultad de Ciencias del Deporte, Universidad de Murcia, C/. Argentina s/n, Santiago de la Ribera, Murcia, España, 30720. E-mail: agranerog@ hotmail.com, abaenaextrem@um.es,mgomezlop@um.es y abraldes@um.es el conocer la satisfacción y diversión que se experimenta en las clases (Baena-Extremera, Granero-Gallegos, BrachoAmador, \& Pérez-Quero, 2012) y por otro el averiguar la importancia que el alumno concede a la asignatura (Gómez-López, Granero-Gallegos, Baena-Extremera, \& Abraldes, en prensa; Moreno, Zomeño, Marín, Ruiz, \& Cervelló, 2013). Para profundizar en el análisis de los distintos factores que influyen en la motivación del estudiante hacia las clases de EF se han utilizado dos de las 
teorías motivacionales más estudiadas en la actualidad. Por un lado, la teoría de la Autodeterminación (Deci \& Ryan, 1985; Ryan \& Deci, 2000) y, por otro, la teoría de las Metas de Logro (Nicholls, 1984, 1989).

La teoría de la Autodeterminación se centra en cómo la motivación intrínseca influye en los personas. Esta teoría se entiende como un continuo en el que se establecen diferentes niveles de autodeterminación, correspondiendo el mayor grado de autodeterminación a las conductas intrínsecamente motivadas. Aquel estudiante que participa en clase porque disfruta aprendiendo y vivenciando las diferentes prácticas, reflejaría una motivación intrínseca (González-Cutre, Sicilia, \& Moreno, 2011), mientras que aquel que practica por razones externas, como por ejemplo practicar debido al conocimiento de los beneficios de esta asignatura, por culpabilidad o por evitar un castigo, ostenta una motivación extrínseca (González-Cutre et al., 2011). Finalmente, quien no se encuentra motivado ni intrínseca ni extrínsecamente (Pelletier, Vallerand, Green-Demers, Brière, \& Blais, 1995), es decir, no tiene la intención de realizar algo (Ryan \& Deci, 2000), se encuentra amotivado. Este alumnado considera la $\mathrm{EF}$ como una pérdida de tiempo (González-Cutre et al., 2011).

Por otro lado, en la Teoría de las Metas de Logro, Nicholls (1989) estableció la existencia de dos orientaciones para demostrar habilidad o formas de concebir el éxito: la orientación a la tarea y la orientación al ego. De tal manera, cuando un estudiante presenta una orientación hacia la tarea dirige su meta al aprendizaje y juzga su nivel de capacidad consigo mismo; mientras que cuando se orienta al ego, la meta se caracteriza por la competitividad y juzga sus competencias comparándose con el resto (Nicholls, 1984).

En esta investigación se ha optado por el Perception of Success Questionnaire (POSQ) diseñado por Roberts y Balagué (1991) y Roberts, Treasure y Balagué (1998). Este instrumento ha sido utilizado y adaptado en diversos estudios relacionados con la EF a nivel internacional, demostrando ser un instrumento valido y fiable. En Inglaterra, Wang, Chatzisarantis, Spray, y Biddle (2002) analizaron 427 estudiantes de entre 11 y 14 años, obteniendo una consistencia interna de .79 para la orientación a la tarea y .82 para la orientación al ego. En Estados Unidos, Standage y Treasuse (2002) validaron esta escala a la EF con 318 estudiantes de 12 a 14 años, obteniendo una consistencia interna de .90 en la orientación a la tarea y de .89 en la orientación al ego. En Portugal, Pereira y Bento (2009) la validaron con 538 alumnos, obteniendo una consistencia interna de .90 en la orientación a la tarea y de .89 en la orientación al ego. En España, Cervelló, Escartí y Balagué (1999) desarrollaron la versión en castellano del Cuestionario de Percepción de Éxito en el Deporte, la cual ha sido muy utilizada en el contexto deportivo y referencia para su aplicación en educación. Resaltar que apenas existen trabajos que expongan y apoyen las propiedades psicométricas de la estructura factorial en su adaptación a la EF. Partiendo de la citada versión en español adap- tada al contexto deportivo, Martínez, Alonso y Moreno (2006) expusieron el análisis factorial confirmatorio del instrumento en una muestra de 1126 estudiantes de EF en secundaria. Sin embargo, y como exponen los propios autores, son necesarios más estudios que corroboren o refuten las propiedades psicométricas.

A partir de estos trabajos, y teniendo en cuenta la carencia de estudios que existe sobre las propiedades psicométricas del POSQ en EF, el objetivo de este estudio es doble. En primer lugar, aportar evidencias sobre la dimensionalidad de la versión española del POSQ, a partir de la escala original (children's version) de Roberts et al. (1998), en una muestra de adolescentes estudiantes de educación secundaria mediante procedimientos confirmatorios. Tras explicar el proceso de adaptación de los ítems, se analizaron las propiedades psicométricas de la versión española del POSQ adaptado a la EF; para ello se concretó: (a) examinar su estructura factorial con análisis factorial confirmatorio (AFC), (b) evaluar la consistencia interna mediante alfa de Cronbach, coeficiente de fiabilidad compuesta y varianza media extractada, (c) verificar su estabilidad temporal, y (d) evaluar las diferencias según género y edad. Como segundo objetivo se plantea estudiar la predicción de la importancia y utilidad concedida al EF a partir de las orientaciones de meta y en función de la competencia y satisfacción intrínseca del alumnado.

\section{Método}

\section{Participantes}

En este estudio participaron 846 estudiantes (463 varones, 54.73\%; 363 mujeres, $45.27 \%$ ) de Secundaria de la Región de Murcia. El rango de edad estuvo comprendido entre 12 y 19 años $(M=15.47 ; S D=1.32)$, siendo la edad media de los chicos, $15.44(S D=1.30)$, y la de las chicas, $15.51(S D=1.34)$. Para evaluar la estabilidad temporal del POSQ adaptado a la EF se utilizó una segunda muestra de 147 estudiantes, elegida previamente de forma aleatoria y compuesta por 76 chicos $(M=15.25 ; S D=1.26)$ y 71 chicas $(M=15.46 ; S D=1.31)$ que completaron otra vez el instrumento siete semanas más tarde. Para prevenir posibles tendencias de deseabilidad social los adolescentes fueron instruidos para utilizar su fecha de nacimiento para identificar cuestionarios (Baena-Extremera et al., 2012).

\section{Instrumentos}

Perception of Success Questionnaire (POSQ; Roberts \& Balagué, 1991; Roberts et al., 1998). Se partió de la versión española del instrumento utilizada en deporte (Cervelló et al., 1999). Este instrumento consta de 12 ítems para medir las orientaciones de meta disposicionales en el deporte mediante dos dimensiones que miden la Orientación hacia la tarea (6 ítem) y la Orientación hacia el ego (6 ítems). En las instrucciones se pide a los sujetos que indiquen el grado de acuerdo o desacuerdo con los ítems, recogiéndose las respuestas en una escala politómica de 5 
puntos que oscila desde muy en desacuerdo (1) a muy de acuerdo (5). En el presente estudio, la consistencia interna de la subescala Orientación hacia el ego fue de $\alpha=.90$ y la de Orientación hacia la tarea, $\alpha=.86$. Se recabaron, además, datos socio-demográficos de los participantes.

Escala de las Necesidades Psicológicas Básicas en el Ejercicio (Vlachopoulos \& Michailidou, 2006). Se utilizó el factor "competencia" de la citada escala, adaptada a la EF en el contexto español por Moreno, González-Cutre, Chillón, y Parra (2008). Dicho factor estaba compuesto de 4 ítems que se respondían en una escala de ítems politómicos de 5 puntos que oscila entre 1 (totalmente en desacuerdo) y 5 (totalmente de acuerdo). En este estudio este factor mostró un valor $\alpha=.78$.

Satisfacción Intrínseca en el Deporte (SSI-EF; BaenaExtremera et al., 2012). Se utilizó la versión española del Sport Satisfaction Instrument adaptada a la EF del original Sport Satisfaction Instrument (Balaguer, Atienza, Castillo, Moreno, \& Duda, 1997; Duda \& Nicholls, 1992). El SSIEF consta de 8 ítems para medir la satisfacción intrínseca en una actividad mediante dos subescalas que miden satisfacción/diversión (5 ítems) y aburrimiento (3 ítems). En las instrucciones se pide que se indique el grado de acuerdo con los ítems que reflejan criterios de diversión o aburrimiento, recogiéndose las respuestas en una escala de ítems politómicos de 5 puntos que oscila desde muy en desacuerdo (1) a muy de acuerdo (5). La consistencia interna de la subescala satisfacción/diversión fue de $\alpha=.86$ y la de aburrimiento, $\alpha=.72$.

Importancia y utilidad de la Educación Física (IEF; Moreno, González-Cutre, \& Ruiz, 2009). Se midió la importancia y utilidad concedida por el alumnado a la EF (3 ítems). Los estudiantes responden en una escala de ítems politómicos con rango de puntuación entre 1 (totalmente en desacuerdo) y 4 (totalmente de acuerdo). En este estudio la fiabilidad fue .76 .

\section{Proceso de Adaptación}

Se adaptó la versión española del POSQ (Cervelló et al., 1999), al ser su objeto de estudio el deporte y el nuestro la EF. La evaluación cualitativa de ítems (validez de contenido) se realizó mediante el juicio de cuatro expertos (Osterlind, 1989): dos en construcción de escalas y dos conocedores del constructo a evaluar. El encabezamiento fue: "En las clases de Educación Física siento que tengo éxito...". La nueva versión fue administrada a 60 estudiantes ( 34 chicos y 26 chicas) de secundaria entre 12 y 19 años. Los comentarios de los mismos sobre instrucciones y forma de redacción supusieron cambios menores. Tras el análisis de los resultados psicométricos obtenidos y una última revisión por parte del equipo de investigación se llegó a la versión final española del POSQ adaptado a la EF (ver Anexo).

\section{Procedimiento}

Se obtuvo permiso para realizar la investigación por parte de los órganos competentes, tanto de los centros educativos de secundaria como universitarios. Se informó, en detalle, a padres/tutores y adolescentes acerca del protocolo y objeto del estudio. La firma del consentimiento informado por parte de ambos fue requisito indispensable para poder participar. Los instrumentos para medir las diferentes variables se administraron en el aula, en presencia de un encuestador y sin la presencia del docente. Cada participante tuvo 10-20 minutos para completar los cuestionarios. Se respeta el anonimato en las respuestas.

\section{Análisis Estadísticos}

Dado que las estructuras que subyacen al instrumento analizado han sido consistentemente determinadas en la literatura, para evaluar la estructura factorial de la escala se realizó un análisis factorial confirmatorio (AFC) con LISREL. Los análisis de ítems, homogeneidad y estructura interna, correlación y consistencia interna de la escala, así como las pruebas para determinar las diferencias de género y edad (MANOVA), se realizaron con el SPSS 17.0. Para estudiar la predicción de la importancia y utilidad concedida por el alumnado a la EF según las orientaciones de meta, competencia y la satisfacción en EF se utilizó un Modelo de Ecuaciones Estructurales (SEM) realizado con LISREL 8.80

\section{Resultados}

\section{Descripción Inicial de los Datos}

En la Tabla 1 se muestran los estadísticos descriptivos del POSQ. En el análisis estadístico de ítems se mantuvo la distribución ítem-factor observada en el instrumento original (Cervelló et al., 1999; Roberts \& Balagué, 1991; Roberts et al., 1998). Las características de los ítems fueron analizadas comprobando si el alfa de la escala aumentaba con la eliminación del algún ítem, así como teniendo en cuenta los criterios apuntados por Nunnally y Bernstein (1995) para conservar un ítem dentro de un factor: coeficiente de correlación corregido ítem-total (CCIT-c) $\geq .30$, desviación típica $(S D)>1$, y que todas las opciones de respuesta habían sido usadas en algún momento. Como se puede comprobar, y según las recomendaciones de Bollen y Long (1994), los índices de asimetría y curtosis son próximos a 0 y $<2$ en las dos subescalas.

Los ítems del primer factor (Orientación al ego) presentaron valores medios entre 2.90 para el ítem 12 y 3.24 para el ítem 9. Las $S D$ fueron $>1$, oscilando entre 1.31 de los ítems 5 y 9 y 1.41 del ítem 12 . La consistencia interna de esta dimensión fue adecuada $(\alpha=.90)$. Todos los CCIT-c presentaron valores $>.63$. El segundo factor (Orientación a la tarea) presentó ítems con valores medios entre 4.03 para el ítem 3 y 4.16 del ítem 8 . Las $S D$ fueron $>1$, oscilando entre 1.04 (ítem 8 y 7) y 1.11 (ítem 10 y 11). La consistencia interna de esta dimensión fue adecuada $(\alpha=.86)$. Todos los CCIT-c presentaron valores >.62. Además, algunos autores, como Carretero-Dios y Pérez (2007), recomiendan realizar un estudio de correlación 
para asegurar la homogeneidad de cada dimensión. En el presente trabajo, la correlación entre la puntuación de cada ítem y la puntuación total en cada uno de los compo- nentes muestran correlaciones positivas con la dimensión teórica a la que pertenecen; asimismo, aunque presentan correlaciones positivas con la puntuación total de la otra dimensión, son valores bajos $(<.23)$.

Tabla 1

Estadísticos Descriptivos, de Consistencia Interna y de Homogeneidad

\begin{tabular}{lccccccc}
\hline Escala: & $M$ & $S D$ & CCIT-c & CC & $\alpha$ sin ítem & Asimetría & Curtosis \\
\hline Orientación al ego $(\alpha=.90)$ & & & & & & & \\
Ítem 12 & 2.90 & 1.41 & .75 & .15 & .88 & .07 & -1.24 \\
Ítem 5 & 3.01 & 1.31 & .74 & .22 & .87 & .00 & -1.01 \\
Ítem 2 & 2.97 & 1.37 & .77 & .21 & .87 & -.08 & -1.16 \\
Ítem 6 & 2.91 & 1.38 & .74 & .21 & .88 & .06 & -1.19 \\
Ítem 1 & 2.65 & 1.40 & .68 & .16 & .89 & .30 & -1.18 \\
Ítem 9 & 3.24 & 1.31 & .64 & .20 & .89 & -.18 & -1.04 \\
Orientación a la tarea $(\alpha=.86)$ & & & & & & & \\
Ítem 8 & 4.16 & 1.04 & .69 & .10 & .83 & -1.11 & .38 \\
Ítem 4 & 4.06 & 1.08 & .64 & .19 & .84 & -1.04 & .51 \\
Ítem 7 & 4.08 & 1.04 & .64 & .17 & .84 & -1.05 & .47 \\
Ítem 11 & 4.06 & 1.11 & .63 & .18 & .84 & -1.08 & .31 \\
Ítem 10 & 3.96 & 1.11 & .65 & .22 & .83 & -.84 & .00 \\
Ítem 3 & 4.03 & 1.08 & .64 & .20 & .84 & -1.08 & .60 \\
\hline
\end{tabular}

Nota. M: media; SD: desviación típica; CCIT-c: coeficiente de correlación corregido ítem-total; CC: correlación de cada ítem con la otra dimensión.

\section{Análisis Factorial Confirmatorio}

Con el objeto de confirmar la dimensionalización original propuesta teóricamente, se aplicaron modelos de ecuaciones estructurales. La estructura factorial del instrumento se evaluó con AFC, utilizando el método de estimación "weighted least squares" (WLS) para variables ordinales del programa LISREL 8.80 (Jöreskog \& Sörbon, 1993). Como input para el análisis de datos se utilizó la matriz de correlaciones policóricas y la matriz de covarianzas asintóticas. Se hipotetizó un modelo de medida, consistente en un modelo de dos factores que asumió la existencia de dos variable latentes. Por ello, el ajuste del modelo fue evaluado con una combinación de índices de ajuste absolutos y relativos (Bentler, 2007). Entre los absolutos, se utilizó el valor p asociado con el estadístico chi cuadrado $\left(\chi^{2}\right)$, que prueba el modelo nulo frente al modelo hipotetizado, con valores de $p>.05$ (Hooper, Coughlan, $\&$ Mullen, 2008). La ratio entre $\chi^{2}$ y grados de libertad $\left(g l ; \chi^{2} / g l\right)$ es un heurístico que se utiliza para reducir la sensibilidad del $\chi^{2}$ al tamaño de la muestra.; Hu y Bentler (1999) consideran aceptables valores $<5.0$. Además, se ha calculado el GFI (índice de bondad de ajuste); autores como Hooper et al. (2008) consideran que el valor debe ser $\geq .95$. Entre los índices relativos: NFI (índice de ajuste normalizado), NNFI (índice de ajuste no normativo), y CFI (índice de ajuste comparativo). En los índices incrementales se considera que valores $\geq .95$ indican un buen ajuste (Hu \& Bentler, 1999). Autores como Kline (2005) recomiendan la utilización de RMSEA (error de aproximación cuadrático medio) y, según Hu y Bentler (1999) un valor $\leq .06$ indicaría un buen ajuste. Los parámetros estimados se consideran significativos cuando el valor asociado al $t$-value $>1.96(p<.05)$.

Los índices de bondad de ajuste obtenidos en el modelo presentado mostraron un muy buen ajuste: $\chi^{2}=124.29, \mathrm{gl}$ $=53, p<.001, \chi^{2} / g l=2.34$, GFI $=.98, \mathrm{NFI}=.97$, NNFI $=.98, \mathrm{CFI}=.98, \mathrm{RMSEA}=.04$. Todos los ítems mostraron valores de fiabilidad individual $\left(R^{2}\right)$ superiores a .05 . Asimismo, el modelo presenta los requisitos mínimos para poder garantizar la validez convergente del modelo (Hair, Black, Babin, \& Anderson, 2009): cargas factoriales estandarizadas elevadas ( $>.60$ en todos los casos) y estadísticamente significativas ( $t$-value $>1.96)$. 


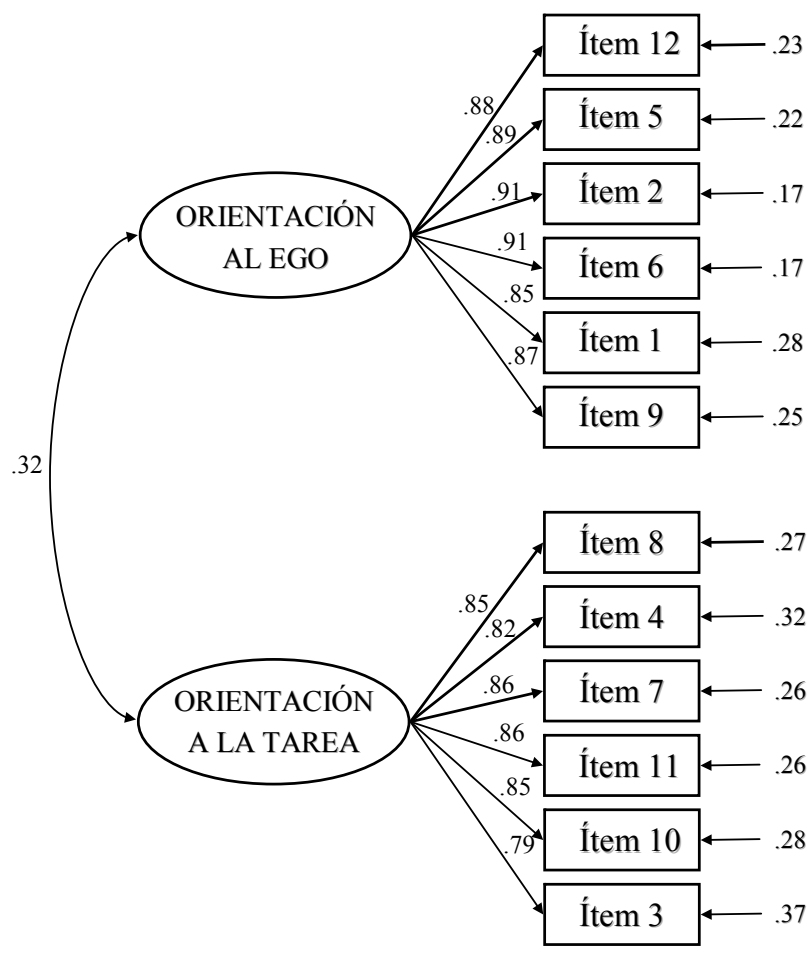

Figura 1. Path diagram del AFC, con pesos estandarizados y errores de medición de cada uno de los ítems de la versión española del POSQ adaptado a la EF.

También es importante, en el AFC de las escalas con naturaleza ordinal de la matriz de correlaciones de datos, ofrecer los resultados de fiabilidad compuesta y varianza media extraída (AVE - Average Variante Extracted) para cada una de las dimensiones críticas (Elosua \& Zumbo,
2008). Según Hair et al. (2009) la fiabilidad compuesta ha tener un valor mínimo de .70 y la AVE de .50. En este trabajo, en ambas subescalas del POSQ, la fiabilidad compuesta fue de .78 y la AVE de .95 .

La estabilidad temporal se evaluó con la muestra de 147 estudiantes que completaron el POSQ en dos ocasiones con intervalo de siete semanas. Los resultados del pretest en Orientación al ego fueron $\alpha=.88$ y en Orientación a la tarea, $\alpha=.83$. Los datos del postest en Orientación al ego fueron $\alpha=.90$ y en Orientación a la tarea, $\alpha=.86$. Los valores de correlación test-retest en la dimensión Orientación al ego fueron $r=.82$ y en Orientación a la tarea, $r=.75$.

\section{Diferencias por Género y Edad}

Para analizar las diferencias en las dos subescalas se realizó un análisis de varianza multivariado (MANOVA $2 \times 3$ ) en el que el género y la edad fueron las variables independientes y la Orientación al ego y la Orientación a la tarea las dependientes. La homogeneidad de la covarianza se examinó mediante el test $\mathrm{M}$ de Box; dado que no resultó significativa ( $\mathrm{M}$ de Box $=21.55, F=1.42, p>.05$ ) se utilizó Lamba de Wilks para evaluar la significación multivariada de efectos principales y de las interacciones. El contraste multivariado no demostró diferencias significativas y efectos de interacción entre las dos variables independientes (género $\mathrm{x}$ edad; Lamba de Wilks $=.99 ; F_{(4,1448)}=.33 ; p>$ $.05)$. Se encontraron diferencias significativas según el género (Lambda de Wilks=.91; $F_{(2,724)}=33.42 ; p<.001$ ), pero no en función de la edad (Lambda de Wilks $=.99$; $\left.F_{(4,1448)}=1.14 ; p>.05\right)$. Las pruebas de los efectos intersujetos mostraron diferencias significativas según género en la Orientación al ego; las chicas presentaron valores por encima de los varones (Tabla 2)

Tabla 2

Análisis Multivariante según Género y Edad

\begin{tabular}{|c|c|c|c|c|c|c|c|c|c|c|c|c|c|c|}
\hline & \multicolumn{4}{|c|}{ Género } & \multicolumn{10}{|c|}{ Edad } \\
\hline & \multicolumn{2}{|c|}{$\begin{array}{l}\text { Varones } \\
(n=463)\end{array}$} & \multicolumn{2}{|c|}{$\begin{array}{l}\text { Mujeres } \\
(n=363)\end{array}$} & \multirow[b]{2}{*}{$F$} & \multirow[b]{2}{*}{$p$} & \multicolumn{2}{|c|}{$\begin{array}{c}12-14 \\
(n=143)\end{array}$} & \multicolumn{2}{|c|}{$\begin{array}{c}15-16 \\
(n=411)\end{array}$} & \multicolumn{2}{|c|}{$\begin{array}{c}17-19 \\
(n=292)\end{array}$} & \multirow[b]{2}{*}{$F$} & \multirow[b]{2}{*}{$p$} \\
\hline & $M$ & $S D$ & $M$ & $S D$ & & & $M$ & $S D$ & $M$ & $S D$ & $M$ & $S D$ & & \\
\hline \multicolumn{15}{|l|}{ POSQ } \\
\hline Orientación al ego & 2.64 & 1.09 & 3.34 & 1.05 & 66.31 & .001 & 2.93 & 1.25 & 2.97 & 1.09 & 2.98 & 1.09 & .02 & .980 \\
\hline Orientación a la tarea & 4.06 & .82 & 4.14 & .79 & 1.81 & .179 & 4.18 & .71 & 4.10 & .84 & 4.02 & .83 & 2.05 & .129 \\
\hline
\end{tabular}

Nota. $M$ : media; $S D$ : desviación típica; $p$ es significativo al valor $<.05$.

\section{Modelo de Ecuaciones Estructurales (SEM)}

La validez empírica del instrumento se evaluó examinando la relación de las dimensiones del POSQ con competencia, SSI-EF e IEF. Para estudiar las propiedades psicométricas de la dimensionalización original propuesta teóricamente en cada uno de los instrumentos utilizados se utilizó el método WLS de LISREL. En la escala de tres variables (IEF), como indican Jöreskog y Sörbom (1993), para el AFC se fijó la varianza del error de una de las variables observadas. Los índices de ajuste obtenidos fueron los siguientes: Competencia: $\chi^{2}=9.75, \mathrm{gl}$ $=2, p<.007, \chi^{2} / g l=4.87, \mathrm{GFI}=.99, \mathrm{NFI}=.98, \mathrm{NNFI}$ 
$=.96, \mathrm{CFI}=.99, \mathrm{RMSEA}=.06 ; \mathrm{SSI}-\mathrm{EF}: \chi^{2}=88.58, g l$ $=19, p<.001, \chi^{2} / g l=4.66, \mathrm{GFI}=.99, \mathrm{NFI}=.96, \mathrm{NNFI}$ $=.95, \mathrm{CFI}=.97$, RMSEA $=.06$; IEF $: \chi^{2}=3.15, g l=1, p$ $<.009, \chi^{2} / g l=3.15, \mathrm{GFI}=.99, \mathrm{NFI}=.99$, NNFI $=.97$, $\mathrm{CFI}=.99, \mathrm{RMSEA}=.02$. Seguidamente, con el objeto de estudiar la predicción de la importancia y utilidad concedida por el alumnado a la EF según las orientaciones de meta, competencia y satisfacción intrínseca, se utilizó un SEM. Por la falta de normalidad de los datos, este análisis se realizó utilizando el método de estimación WLS para variables ordinales del programa LISREL 8.80
(Jöreskog \& Sörbon, 1993). La matriz de correlaciones policóricas y la matriz de covarianzas asintóticas fueron utilizadas como input para el análisis de los datos. En este caso, también se utilizaron diferentes índices de ajustes del modelo. Los resultados obtenidos por el modelo que se presenta (Figura 2) fueron: $\chi^{2}=630.51, g l=225, p$ $<.001, \chi^{2} / g l=2.80, \mathrm{GFI}=.96, \mathrm{NFI}=.96, \mathrm{NNFI}=.98$, $\mathrm{CFI}=.96$, RMSEA $=.06$. Los datos mostraron que el modelo de medición era adecuado. Los datos ofrecidos en la Figura 2, muestran 6 variables latentes con un total de 27 variables observadas.

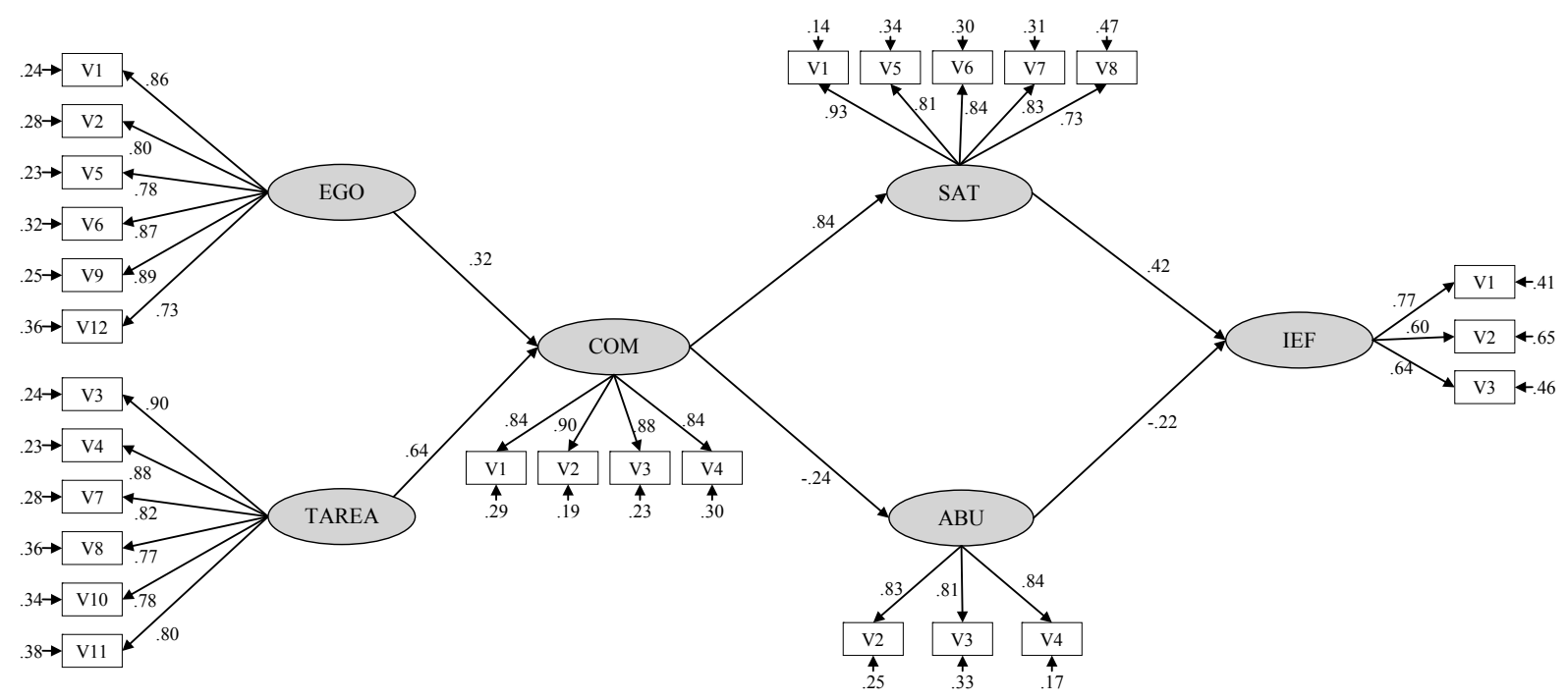

Figura 2. Modelo estructural compuesto por 6 factores hipotetizados. Los círculos representan los constructos latentes y los cuadrados las variables medidas. Todos los parámetros son estandarizados y significativos en $p<.05$

En la Figura 2 se observa una relación de predicción más alta entre la orientación a la tarea y la competencia (.64) que entre la orientación al ego y la competencia (.32). A continuación, la competencia predice positivamente la satisfacción/diversión del estudiante (.84), mientras que lo hace de forma negativa con el aburrimiento (-.24). Seguidamente, es la satisfacción/diversión la que predice de forma positiva la importancia (.42) de la EF.

\section{Discusión y Conclusiones}

Este trabajo ha tenido un doble objetivo: analizar las propiedades psicométricas del POSQ adaptado a EF en una muestra española y estudiar la predicción de la importancia y utilidad concedida a la EF según las orientaciones de meta, percepción de competencia y satisfacción intrínseca del alumnado. La versión española del POSQ ha mostrado la misma distribución factorial que el resto de versiones existentes (Cervelló et al., 1999; Cervelló \& Santos-Rosa, 2000; Escartí, Roberts, Cervelló, \& Guzmán, 1999; Martínez et al., 2006). La fiabilidad de dicho cuestionario a través del alfa de Cronbach ha sido demostrada en España en las clases de EF (Cervelló \& Santo-Rosa, 2000; Moreno,
Conte, Hellín, Hellín, Vera, \& Cervelló, 2008), con alfas de .88 a .80 para la tarea y de .94 a .73 para la subescala ego. En el presente estudio, la consistencia interna de los dos factores muestra claramente que ambos obtuvieron valores sustancialmente por encima del valor mínimo de .70 propuesto por Nunnally y Bernstein (1995). Asimismo, siguiendo las indicaciones de Elosua y Zumbo (2008), junto al alfa de Cronbach, se han añadido evidencias de fiabilidad compuesta y AVE que en ningún otro trabajo en español se han aportado. Los valores de fiabilidad compuesta y de AVE que hemos hallado son claramente superiores a los mínimos requeridos en la literatura (Hair et al., 2009).

En cuanto a los índices de bondad de ajuste, indicar que entre los índices absolutos los datos de $\chi^{2}$, así como la ratio $\chi^{2} / g l$, indican un mejor ajuste que el modelo presentado por Martínez et al. (2006). En el modelo de este trabajo la ratio $\chi^{2} / g l$ es de $2.34,<5.0$ e indicativa de un aceptable ajuste del modelo (Hu \& Bentler, 1999), mientras que en el estudio anterior este índice era de 6.0. Asimismo, los índices FIT reportan mejores resultados, con valores cercanos a 1 y >.95. Aunque autores como Hooper et al. (2008) recomiendan tener más en cuenta la no significatividad del 
chi cuadrado a la hora de decidir aceptar o no un modelo, en una muestra de las características de este estudio, lo normal es que la chi cuadrado resulte significativo (Hair et al., 2009), por lo que se han de atender otros índices, tal y como se ha hecho.

Otro aspecto que fortalece los resultados de esta investigación, es que la escala POSQ ha sido trabajada como consta originalmente (Roberts et al., 1998; Treasure \& Roberts, 1994a, 1994b); es decir, los valores oscilaban de 1 a 5 coincidiendo así con otros trabajos (Pereira \& Bento, 2009; Standage \& Treasure, 2002), al contrario de lo realizado en España hasta el momento (Cervelló et al., 1999; Cervelló \& Santos-Rosa, 2000; Martínez et al., 2006) en la que la escala likert reflejaba un rango de respuesta de 0 a 100. De igual manera, y dados los cambios educativos en materia de lenguaje en España en los últimos años debiendo considerarse la utilización de un lenguaje más coeducativo tanto a nivel hablado como escrito-, se ha trabajado en adaptar los ítems de la escala a una redacción en la que tanto varones como mujeres se vean reflejados, y no solamente los chicos.

Tal y como se puede apreciar en los resultados, la orientación a la tarea predice principalmente la competencia del alumnado. Cuando existe una orientación a la tarea, los sujetos utilizan fuentes internas para juzgar su competencia, de tal forma que consideran que el esfuerzo es una de las causas de éxito en la actividad (Cervelló et al., 1999). Este patrón conlleva que cuando un alumno o alumna obtiene un fracaso en sus resultados, responden con un incremento del esfuerzo y con una mayor persistencia en el desempeño de la actividad (Van Yperen, \& Duda, 1999). Diferentes estudios realizados en educación han demostrado que los sujetos orientados a la tarea tienden a divertirse con la práctica de actividades físicas en clase (Duda \& Nicholls, 1992; Nicholls, 1989). En cambio, con una orientación al ego, se cree que el éxito en la tarea se alcanza a través de la demostración de mayor capacidad y del uso de técnicas engañosas. Normalmente, este alumnado busca tener una mayor aprobación social, conllevando por tanto una menor diversión con la práctica deportiva (Cervelló et al., 1999).

Por último, se observa como la satisfacción/diversión predice positivamente la importancia a la EF, al contrario del aburrimiento. Estudios como los desarrollados por Granero-Gallegos, Baena-Extremera, Pérez-Quero, Ortiz-Camacho, Bracho-Amador (2012) y Moreno et al. (2009), han demostrado que el alumnado de secundaria presentaba una alta motivación intrínseca hacia las clases de EF y sentían satisfacción y diversión con las clases que recibían. Ntoumanis (2005) afirma que cuando un alumno se divierte tiende a estar intrínsecamente motivado y le concede mayor importancia a la asignatura.

Como conclusión final, se puede afirmar que el POSQ tiene una consistencia interna fiable y una estructura factorial estable, por lo que se le considera como un instrumento válido para medir la orientación de meta disposicional en EF. Asimismo, que la orientación a la tarea es la que más influye en la percepción de competencia y esta en la satisfacción /diversión, lo que predice que el alumnado considere más importante y útil la asignatura.

\section{Referencias}

Baena-Extremera, A., Granero-Gallegos, A, Bracho-Amador, C., \& Pérez-Quero, F. J. (2012). Versión española del Sport Satisfaction Instrument (SSI) adaptado a la Educación Física. Revista de Psicodidáctica, 17(2), 377-396.

Balaguer, I., Atienza, F. L., Castillo, I., Moreno, Y., \& Duda, J. L. (1997). Factorial structure of measures of satisfaction/interest in sport and classroom in the case of Spanish adolescents. In Abstracts of $4^{\text {th }}$ European Conference of Psychological Assessment (p. 76). Lisbon, Portugal: European Association of Psychological Assessment.

Bentler, P. M. (2007). On tests and indices for evaluating structural models. Personality and Individual Differences, 42, 825-829.

Bollen, K. A., \& Long, J. (1994). Testing structural equation models. Newbury Park, CA: Sage.

Carretero-Dios, H., \& Pérez, C. (2007). Standards for the development and the review of instrumental studies: Considerations about test selection in psychological research. International Journal of Clinical and Health Psychology, 7, 863-882.

Cervelló, E. M., Escartí, A., \& Balagué, G. (1999). Relaciones entre la orientación de metas disposicional y la satisfacción con los resultados deportivos, las creencias sobre las cusas de éxito en deporte y la diversión con la práctica deportiva. Revista de Psicología del Deporte, 8, 7-19.

Cervelló, E. M., \& Santos-Rosa, F. J. (2000). Motivación en las clases de Educación Física: un estudio de la perspectiva de las metas de logro en el contexto educativo. Revista de Psicología del Deporte, 9, 51-70.

Deci, E. L., \& Ryan, R. M. (1985). Intrinsic motivation and self-determination in human behaviour. New York: Plenum.

Duda, J. L., \& Nicholls, J. G. (1992). Dimensions of achievement motivation in scholwork and sport. Journal of Educational Psychology, 84, 290-299.

Elosua, P., \& Zumbo, B. D. (2008). Coeficientes de fiabilidad para escalas de respuesta categórica ordenada. Psicothema, 20(4), 896-901.

Escartí, A., Roberts, G. C., Cervelló, E. M., \& Guzmán, J. F. (1999). Adolescents goal orientations and the perception of criteria of success used by significant others. International Journal of Sport Psychology, 30, 309-324.

Gómez-López, M., Granero-Gallegos, A., Baena-Extremera, A., \& Abraldes, J. A. (en prensa). Análisis de los perfiles motivacionales y su relación con la importancia de la educación física en secundaria. Revista Iberoamericana de Diagnóstico y Evaluación Psicológica, 36.

González-Cutre, D., Sicilia, A., \& Moreno, J. A. (2011). Un estudio cuasi-experimental de los efectos del clima motivacional tarea en las clases de educación física. Revista de Educación, $356,677-700$.

Granero-Gallegos, A., Baena-Extremera, A., Pérez-Quero, F. J., Ortiz-Camacho, M. M., \& Bracho-Amador, C. (2012). Analysis of motivational profiles of satisfaction and importance of physical education in high school adolescents. Journal of Sports Science and Medicine, 11, 614-623.

Hair, J. F., Black, W. C., Babin, B. J., \& Anderson, R. E. (2009). Multivariate data analysis ( $7^{\text {th }}$ ed.). New York: Pearson Prentice Hall. 
Hooper, D., Coughlan, J., \& Mullen, M. (2008). Structural equation modelling: Guidelines for determining model fit. Electronic Journal of Business Research Methods, 6(1), 53-60.

$\mathrm{Hu}$, L., \& Bentler, P. M. (1999). Cutoff criteria for fit indexes in covariance structure analysis: Conventional criteria versus new alternatives. Structural Equation Modelling, 6, 1-55.

Jöreskog, K. G., \& Sörbom, D. (1993). Structural equation modeling with the SIMPLIS command language. Chicago, IL: Scientific Software International.

Kline, R. B. (2005). Principles and practice of structural equation modeling ( $2^{\text {nd }}$ ed.). New York: The Guilford Press.

Martínez, C., Alonso, N., \& Moreno, J. A. (2006). Análisis factorial confirmatorio del "Cuestionario de Percepción de Éxito (POSQ)" en alumnos adolescentes de Educación Física. In M. A. González, J. A. Sánchez, \& A. Areces (Eds.), IV Congreso de la Asociación Española de Ciencias del Deporte (pp. 757761). A Coruña, España: Xunta de Galicia.

Moreno, J. A., Conte, L., Hellín, P., Hellín, G., Vera, J. A., \& Cervelló, E. (2008). Predicción de la motivación autodeterminada según las estrategias para mantener la disciplina y la orientación motivacional en estudiantes adolescentes de educación física. Apuntes de Psicología, 26(3), 501-516.

Moreno, J. A., González-Cutre, D., Chillón, M., \& Parra, N. (2008). Adaptación a la educación física de la escala de las necesidades psicológicas básicas en el ejercicio. Revista Mexicana de Psicología, 25(2), 295-303.

Moreno, J. A., González-Cutre, D., \& Ruiz, L. M. (2009). Selfdetermined motivation and physical education importance. Human Movement, 10(1), 5-11.

Moreno, J. A., Zomeño, T. E., Marín, L. M., Ruiz, L. M., \& Cervelló, E. (2013). Percepción de la utilidad e importancia de la EF según la motivación generada por el docente. Revista de Educación, 362.

Nicholls, J. G. (1984). Achievement motivation: Conceptions of ability, subjective experience, task choice, and performance. Psychological Review, 91, 328-346.

Nicholls, J. G. (1989). The competitive ethos and democratic education. Cambridge, MA: Harvard University Press.

Ntoumanis, N. (2005). A prospective study of participation in optional school physical education using a self-determination theory framework. Journal of Educational Psychology, 97, 444-453.

Nunnally, J. C., \& Bernstein, I. J. (1995). Teoría psicométrica. Madrid, España: Mcgraw-Hill.

Osterlind, S. J. (1989). Constructing test items. London: Kluwer Academic.

Pelletier, L. G., Vallerand, R. J., Green-Demers, I., Brière, N. M., \& Blais, M. R. (1995). Leisure and mental health: Relationship between leisure involvement and psychological well-being. Canadian Journal of Behavioural Science, 27, 214-225.

Pereira, P., \& Bento, M.F. (2009). Questionário de Percepção de Êxito em Educação Física: Estudo de validação no contexto português. In Actas do X Congresso Internacional GalegoPortuguês de Psicopedagogia (pp. 4650-4662). Braga, Portugal: Universidade do Minho.

Roberts, G. C., \& Balagué, G. (1991). The development and validation of the Perception of Success Questionnaire. Paper presented at the European Federation of Sport Psychology Congress, Cologne, Germany.

Roberts, G. C., Treasure, D. C., \& Balagué, G. (1998). Achievement goals in sport: The development and validation of the Perception of Success Questionnaire. Journal of Sport Sciences, 16, 337-347.
Ryan, R. M., \& Deci, E. L. (2000). Self-determination theory and the facilitation of intrinsic motivation: Social development and well-being. American Psychologist, 55, 68-78.

Standage, M., \& Treasure, D. C. (2002). Relationship among achievement goal orientations and multidimensional situational motivation in physical education. British Journal of Educational Psychology, 72, 87-103.

Treasure, D. C., \& Roberts, G. C. (1994a). Cognitive and affective concomitants of task and ego goal orientations during the middle school years. Journal of Sport \& Exercise Psychology, $16,15-28$

Treasure, D. C., \& Roberts, G. C. (1994b). Perception of success questionnaire: Preliminary validation in an adolescent population. Perceptual and Motor Skills, 79, 607-610.

Van Yperen, N., \& Duda, J. (1999). Goal orientations, beliefs about success, and performance improvement among young elite Dutch soccer players. Scandinvian Journal of Medicine and Science in Sports, 9(6), 358-364.

Vlachopoulos, S. P., \& Michailidou, S. (2006). Development and initial validation of a measure of autonomy, competence, and relatedness in exercise: The Basic Psychological Needs in Exercise Scale. Measurement in Physical Education and Exercise Science, 10, 179-201.

Wang, C. K. J., Chatzisarantis, N. L. D., Spray, C. M., \& Biddle, S. J. H. (2002). Achievement goal profiles in school physical education: Differences in self determination, sport ability beliefs, and physical activity. British Journal of Educational Psychology, 72, 433-445. 
Anexo

\section{Versión española del “Perception of Success Questionnaire (POSQ)" adaptado a la Educación Física}

Ítem 1. Cuando derroto a los demás

Ítem 2. Cuando soy el/la mejor

Ítem 3. Cuando trabajo duro

Ítem 4. Cuando demuestro una clara mejoría personal

Ítem 5. Cuando mi actuación supera la del resto de alumnos/as

Ítem 6. Cuando demuestro al/la profesor/a y a mis compañeros/as que soy el/la mejor

Ítem 7. Cuando supero las dificultades

Ítem 8. Cuando domino algo que no podía hacer antes

Ítem 9. Cuando hago algo que los demás alumnos/as no pueden hacer

Ítem 10. Cuando rindo a mi mejor nivel de habilidad

Ítem 11. Cuando alcanzo una meta

Ítem 12. Cuando soy claramente superior 